\title{
laborhifórico
}

ISSN 2359-6910

https://revistas.ufrj.br/index.php/lh/

ARTIGO

Recebido em 17 de novembro de 2020

Aprovado em 12 de fevereiro de 2021

\section{Migración y contacto de lenguas: nuevas variedades y reestructuración del diasistema}

Migration and language contact: new varieties

and restructuring of the diasystem

DOI: https://doi.org/10.24206/lh.v7i1.39522

Klaus Zimmermann

Doctorado en la Universidad de Mannheim. "Habilitación" (segundo doctorado) en la Universidad Libre de Berlín. Profesor catedrático de lingüística iberorrománica de la Universidad de Bremen 1996-2012. Antes profesor en la Universidad Libre de Berlín, en la Universidad Nacional Autónoma de México y director del Departamento de investigación del Instituto Ibero-Americano PK de Berlín. Era fundador y director de la Revista Internacional de Lingüística Iberoamericana y de la serie LENSO (Editorial Iberoamericana). Campos de investigación: el contacto de lenguas amerindias con el español, lengua y migración, lengua e identidad, lingüística pragmática (cortesía), sociolingüística, lenguaje de jóvenes, política y planificación lingüística, lenguas criollas, historia de la lengua española en América, historiografía de la lingüística (lingüística misionera, Wilhelm von Humboldt), análisis de discurso.

E-mail:kzimmermann@uni-bremen.de ORCID: http://orcid.org/0000-0001-6431-9756 


\section{RESUMEN}

El contacto de lenguas en situaciones de migración colectiva y masiva no desemboca directamente en la emergencia de una nueva lengua, tampoco directamente en el desuso inmediato y la pérdida de una de las lenguas afectadas sino en la emergencia de una diversidad de variedades de contacto. La lingüística del contacto de lenguas debe focalizar sistemáticamente esta gama de variedades de contacto. En esta hay variedades más cercanas a la lengua originaria de los migrantes y otras más cercanas a la lengua de la comunidad de recepción. Datos empíricos para esta visión son el "spanglish" y los semicriollos. Tomando el concepto de lengua como diasistema se está desarrollando un diasistema reestructurado y en este sentido nuevo. Los hablantes en situaciones bilingües construyen los factores relevantes que les guían para su comportamiento lingüístico de manera individual. Toman los recursos lingüísticos disponibles como repertorio de expresión sin ocuparse mucho de nociones de pureza. La situación bilingüe revela debilidades de la teoría de diasistemas que se define tradicionalmente como perteneciente a una lengua. El diasistema no debe conceptualizarse como arquitectura de variedades de una lengua sino como marco de referencia debe tomarse la comunidad de comunicación. Por ello se propone una redefinición parcial del concepto de diasistema.

Palabras clave: Variedades de contacto. Translingualización/translanguaging. Semicriollo. Spanglish. Chicanos. Diasistema. Comunidad de comunicación.

\section{ABSTRACT}

Language contact in situations of collective migration does not lead directly to the emergence of a new language, nor directly to the immediate disuse and loss of one of the affected languages, but to the emergence of a diversity of varieties of contact. The linguistics of language contact must systematically try to describe this range of varieties of contact. In this there are varieties closer to the original language of the migrants and others closer to the language of the receiving community. Empirical data for this vision are "Spanglish" and semicreoles. Taking the concept of language as a diasystem, a restructured and in this sense new diasystem is developing. Speakers in bilingual situations construct the relevant factors that guide them for their linguistic behavior individually. They take the linguistic resources available as a repertoire of expression without dealing too much with notions of purity. This is shown in the case of "Spanglish" and semicriollos. The application of 
the theory of diasystem to bilingual situations reveals weaknesses because it is traditionally defined as belonging to one language only. It is argued that the diasystem should not be conceptualized as architecture of varieties of one language but rather the communication community should be taken as the frame of reference. For this reason, a partial redefinition of the concept of diasystem is proposed.

Keywords: Contact varieties. Translanguaging. Semicreole. Spanglish. Chicanos. Diasystem. Communicative community 


\section{Introducción}

Los estudios del contacto lingüístico han focalizado, de preferencia en el pasado, los cambios en el sistema o sea en la estructura fonético-fonológica, gramatical y lexical de las lenguas afectadas. Esto tenía su razón de ser en el axioma de la lingüística estructural de que el objeto de esta ciencia es la lengua (sistema y norma), apoyándose en la bien conocida tripartición saussureana de lenguaje (langage), lengua (langue) y habla (parole). Como contacto se veía solo la influencia de una lengua sobre otra: Se consideraba, dado un contacto, cuándo un elemento o estructura de una lengua era integrado en el sistema de otra. Uriel Weinreich - al cual podemos considerar el fundador de la lingüística de contacto moderna - ha enfocado esta disciplina desde una

"triple perspectiva: 1) lingüística, cuyo objetivo es el análisis de los fenómenos que afectan a los sistemas lingüísticos en concurrencia (...) y cuyo centro gravita en torno al concepto de interferencia lingüística; 2) psicolingüística, cuyo objetivo es el estudio de la problemática del individuo bilingüe y cuyo centro de interés es el concepto de la competencia bilingüe; 3) sociolingüística, cuyo objetivo es el estudio de las comunidades multilingües, centrada en las relaciones de congruencia entre grupos sociales y grupos lingüísticos así como en los efectos del sistema sociocultural sobre el uso lingüístico (Argente 1998: 4-5).

Como dice también Argente, Weinreich se interesa primordialmente por la primera de las tres perspectivas. Weinreich distingue también entre fenómenos de contacto a nivel del habla (parole) y a nivel de la lengua (langue). Los primeros no se han integrado todavía (en el momento del análisis) en el sistema de la lengua. Cabe reconocer hoy que la distinción categorial parece clara; sin embargo, la atribución de los fenómenos observados a cada categoría es muchas veces difícil, si no imposible, y esto se debe al hecho de que el concepto de sistema (colectivo) ${ }^{1}$ y habla (¿individual?) prescinde del sistema individual (idiolecto) y la realidad de pluralidad de los sistemas individuales. Cabe reconocer que en el momento de análisis del proceso de contacto en un grupo (de migrantes) no todos los individuos-hablantes hacen uso de los mismos procedimientos de manejo de las lenguas de contacto (por ejemplo: no tienen el mismo estado de desarrollo de integración de elementos de la lengua ajena en la suya ni de las interferencias de su lengua nativa cuando hablan la lengua ajena). Este es un aspecto importantísimo: ¿Cómo juzgar, entonces, si hay una integración al sistema (transindividual o colectivo) o no? ¿O debemos asumir que se trata de sistemas (y normas) individuales? El reconocimiento central y justo de Uriel Weinreich, de que el contacto tiene lugar en el individuo, el hablante, o como se diría hoy, en el sistema cognitivo del hablante, implica que se trata de un sistema

\footnotetext{
${ }^{1}$ La creencia de un sistema colectivo idéntico es si no una suposición de la observación (igualmente suposición) de la comprensión mutua de los participantes en el intercambio de mensajes lingüísticos.
} 
individual y que tenemos una diversidad y variación de sistemas afectados por las situaciones de contacto, las que también varían para cada individuo o para diversos grupos de los migrantes Sin embargo, la lingüística del contacto tenía como objetivo - describir nuevas lenguas, es decir entidades colectivas. La multitud de variedades individuales, sociales y regionales era considerada tan solo como un paso sin mayor interés para la lingüística, es decir la construcción de lo que se considera como el objeto de esta ciencia, la descripción del sistema (que por definición se considera como colectivo) de una nueva lengua. El trasfondo de este objetivo es el axioma sobreentendido de que haya identidad de los códigos individuales de la comunidad lingüística para que haya posibilidad de comprensión. No obstante, hoy sabemos, que lo que pasa en lo que se llama contacto de lenguas es un proceso (no solo un resultado). Y este aspecto procesual es altamente instructivo: No debe centrarse únicamente en los sistemas colectivos ya fijos como resultado del contacto, sino también en los diversos manejos del material lingüístico de las dos (o más) lenguas por parte de los hablantes. Y se debe tener en cuenta que el entendimiento no es solo una cuestión de los supuestos códigos idénticos, sino que actúa también el esfuerzo cognitivo de entender lo que quiere decir el interlocutor a pesar de que se trata de una estructura nueva con material lingüístico en parte desconocido: es decir la activación de la capacidad de construcción mental de sentido y la percepción del procesamiento nuevo. Y en el futuro el interlocutor oyente podría utilizar este mismo nuevo procedimiento. Este proceso es altamente complejo y variado, sus varias formas dependen de factores culturales, lingüísticos (tipo de lenguas en contacto), condiciones del contacto como tipo de migración (individual, colectiva, definitiva, temporal, económica, personal-relacional, forzada-esclavista, conquistadora) ${ }^{2}$, por la vía del medio oral, escrito, medios masivos de comunicación, aprendizaje anterior o no, circunstancias actitudinales, societales, económicas, políticas y factores valorativos, filosóficos, etc. Hay que distinguir el proceso de contacto individual y el proceso a nivel social o colectivo. Este último es una multitud de procesos diferentes que eventualmente pueden, no necesariamente deben, embocar a una nueva variedad compartida por todos los que están afectados por el contacto. O siquiera pueden emerger diferentes variedades de contacto (sociales, regionales) que se mantienen como tales con sus funciones específicas. Desde el punto de vista de la teoría del contacto de lenguas es importante no desconsiderar estas etapas pasajeras o diferenciación de variedades de contacto, ya que dejan ver las formas diferentes del manejo de lenguas en diferentes situaciones de contacto. Es verdad que en procesos de contacto pasados puede ser difícil encontrar manifestaciones de estas formas en etapas iniciales del contacto por el hecho de que las fases iniciales se hacen preferentemente por la vía oral y hay pocos testimonios escritos o grabados en otros medios. Sin embargo, son estas las que revelan las soluciones diferentes empleadas para hacerse entender con material lingüístico nuevo, incompleto, semiestructurado, mal procesado etc. Los variados microprocesos del contacto como inferencia, transferencia, simplificación, 
etc. son entonces una parte esencial del estudio del contacto, pero hay que integrar a los estudios del contacto, además, otra realidad psico y sociolingüística, la percepción del conjunto de los microprocesos del contacto como otra o como nueva variedad.

\section{La lengua como diasistema de variedades}

Es hoy de aceptación común que una lengua no es un sistema homogéneo: Para muchas entidades de referencia y significado existen variables (alternativas de denominación y expresión). Estas se pueden agrupar bajo criterios extralingüísticos que se llaman variedades; así que lo que llamamos "lengua" (española, alemana, francesa, portuguesa, etc.) existe solo en forma de un conglomerado de variedades. En algunas teorías se llama este conglomerado "diasistema" (Coseriu 1988, 1981: 306). Este es el resultado de una evolución histórica producida por cambios de diversa índole (hechos geográficos, desarrollos económicos, actos políticos, diferenciaciones sociales, religiosas y profesionales, invenciones técnicas, innovaciones intelectuales y cognitivo-creativas, deseos identitarios y otros) $)^{3}$. Por lo tanto, no es un sistema en sí, sino se trata del ensayo de una sistematización ex post por la lingüística. Esta depende de la sistematicidad o no sistematicidad de los factores antes mencionados. Como resultado de una evolución histórica se parece en parte más bien a una estructura anárquica. No sé si el edificio de la "arquitectura de variedades" - como lo llamó Eugenio Coseriu (1988) - tiene una forma elegante: parece más a una de estas catedrales católicas con la mezcla de estilo románico, gótico y la constante adición de elementos de otros estilos como el renacimiento, barroco etc. de siglos posteriores. Tiene estabilidad momentánea pero no fija, y está en constante proceso de cambio y reestructuración. La forma sistemática en la que los lingüistas han presentado los diasistemas (variación diatópica, o sea según el espacio geográfico; diastrática, o sea de la distinción social; diafásica, o sea de situaciones de comunicación; diamésica ${ }^{4}$ o sea del medio de comunicación oral o escrito, por ejemplo ${ }^{5}$ ) es un intento de ordenación teórica. Este esquema, no obstante, oculta en cierta forma su evolución anárquica. Las variedades concretas que existen en una lengua no son las mismas que en otra lengua, las de una época no son las mismas que las de otra ni las mismas en la misma lengua que se habla en diferentes países (el caso de las lenguas pluricéntricas, como son el inglés, el español, el portugués, el alemán, el francés, por ejemplo). Tomando el criterio

\footnotetext{
${ }^{3}$ La dialectología, la dialectología social, la sociolingüística y la lingüística de variedades, la estilística y otras disciplinas deben a estos reconocimientos su objeto de interés.

${ }^{4}$ La denominación "diamésica" no es de Coseriu, tampoco de los que propusieron la variación oral versus escrita (lenguaje de cercanía versus de distancia) como la más fundamental (Koch/Oesterreicher 1985), pero la utilizo aquí porque facilita la manera de hablar sobre el asunto.

${ }^{5}$ Hay otras sistematizaciones más o menos parecidas; no quiero entrar en detalles aquí.
} 
de edad, vemos que en el interior de una sociedad tampoco hay simetría de evolución de variedades. El hecho de que haya en el siglo XX una variedad juvenil (mejor: varias variedades juveniles, de impronta contracultural) de una lengua y no - como una visión simétrica podría suponer - una variedad de este tipo de la tercera edad, se explica por razones psicosociales, no por razones lingüísticas.

Estas propuestas teóricas de variación lingüística se deben a construcciones científicas (o etic) que hay que tener en cuenta para entenderlas bien. Una de estas construcciones es el constructo de la lengua como objeto de la lingüística en vez de la práctica de la comunicación. La construcción de la lengua de hecho es una construcción reduccionista; útil en un momento dado de la historia de la lingüística y útil durante tanto tiempo que muchas veces no somos conscientes de la reducción. Hoy sabemos que la realidad de la comunicación consiste en la combinación de códigos semióticos (comunicación multimodal: gestos, mímica, imago-visual, entonación etc.), pero también en la combinación de diferentes lenguas históricas o de sus variedades. Y con ello entramos en el campo del contacto de lenguas producido por una multitud de hechos y factores que vamos a detallar más adelante. Lo importante es que la comunicación real y lo que pasa en este conjunto como construcción de objeto científico nos abre a otras visiones y descubrimos otros fenómenos que describir y explicar: el procesamiento cognitivo de las lenguas o elementos de las lenguas, la construcción de lo propio y lo ajeno, la construcción de lo puro versus lo híbrido, la construcción de espacios adecuados para el uso de una lengua versus otra, de espacios de legitimidad o rechazo del uso de translingualizaciones/translanguaging (de interferencias, transferencias, alternancia de lenguas/code switching), la transgresión de fronteras y reglas (empíricas) lingüísticas y su lenta aceptación.

Hay otro aspecto problemático del esquema clásico de la formulación del concepto de diasistema (sea de formulación coseriana (1986) o hallidayana (1978: 35)): Ha sido elaborado para sus registros disponibles en una lengua, su marco de consideración es una lengua (para una comunidad de hablantes monolingües). Si enfocamos la práctica comunicativa de los hablantes de comunidades migrantes-bilingües, se presenta otra situación: Aumentan sus registros disponibles y aumenta la complejidad del diasistema. Tal vez también debe cambiar el marco teórico, como discutiremos en adelante, tomando como ejemplo la comunidad chicana de los EE.UU.

\section{La perspectiva emic de la variación diatópica: ¿construcción de espacio o construcción de situaciones comunicativas?}

En la teoría de los diasistemas se utilizan las categorías espacio geográfico, estrato y grupo social, situación comunicativa y uso de medio. Primero quiero señalar o recordar que estas categorías - para 
ser cognitivamente pertinentes - deben ser construidas cognitivamente como tales y deben ser atribuidas a un impacto lingüístico. O la construcción de diferencias lingüísticas se correlaciona (también un resultado de construcción) simultáneamente con otros factores para ubicarlas y darles su significado lectal. En segundo lugar, propongo considerarlas como espacios.

Las definiciones de estas categorías - a pesar de sus conocidos criterios - no están del todo suficientemente esclarecidas. Me refiero a su perspectiva de valor. Si la definición de variedad diatópica es el espacio geográfico, es decir un espacio que se entiende inicialmente inferior al espacio de la lengua (histórica), ${ }^{6}$ se asume una perspectiva de correlación de ocurrencias de rasgos lingüísticos con un área desde la perspectiva etic (del observador exterior) para el objetivo de trazar fronteras de su uso y para la comparación con otras variedades diatópicas. La categoría tiene un estatus más bien estático que sugiere que todos los hablantes en el área utilizan la variedad en este espacio (o por lo menos conocen esta variedad y no conocen otra). No se considera la perspectiva emic, es decir la de los hablantes, de las personas involucradas que utilizan esta variedad. Esta perspectiva emic trata de describir la construcción mental de la situación comunicativa y de los criterios que conllevan al hablante de utilizar una y no otra variedad o de mezclar su uso. Este punto de vista asume un enfoque que trata de describir la competencia comunicativa (y lingüística) del interlocutor y sus criterios (muchas veces inconscientes ${ }^{7}$ ) de adecuación, los que le guían a utilizar la variedad idónea para tal situación. En los espacios estables todos conocen la misma variedad y la utilizan y por ello parece como regla que se hace uso siempre de la misma variedad local/regional. Considerar este último aspecto implica la suposición de que el hablante tiene a su disposición por lo menos una variedad alternativa (lo que no era válido para todos los hablantes en todas las regiones). Las fronteras geográficas de la variedad diatópica y el espacio de su uso se constituyen entonces por la percepciónconstrucción de situaciones comunicativas, y estas por una multitud de personas de manera similar. La concienciación de este espacio y de sus fronteras se produce solamente en el momento en que la construcción habitual no se revela adecuada (perspectiva emic) lo que significa la percepción de la transgresión de una frontera (perspectiva etic). Es por ello que los estudios dialectales hechos por medio de grabaciones del habla no dirigidas manifiestan heterogeneidad o polimorfismo de la variedad tanto entre diferentes locutores como también del mismo locutor. ${ }^{8}$ La perspectiva emic de situaciones comunicativas incluye desde el inicio una visión dinámica y de posibles cambios. Especialmente en los casos de la confrontación de varias variedades, sean otros dialectos, sea la lengua estándar, sea otra lengua histórica o una variedad de ella.

\footnotetext{
${ }^{6}$ No hay que olvidar que también la lengua (histórica) tiene un espacio geográfico frente al de otras lenguas.

${ }^{7}$ Pueden concienciarse post festum, pero en general son inconscientes en la situación de uso; cf. la teoría del impacto de la "mano invisible" en el cambio lingüístico (Keller 2003).

${ }^{8}$ Tales resultados de polimorfismo han surgido en los estudios para el Atlas lingüístico de México bajo la dirección de Juan M. Lope Blanch (1990-1992).
} 
Cuando se ha construido el concepto de diasistema diatópico los constructores tenían como realidad enfocada a la lengua alemana, italiana y española (en España). Sin embargo, la caracterización diatópica de las lenguas pluricéntricas es mucho más complicada. También las variedades nacionales de España, México, Argentina, Cuba etc. son variedades diatópicas que alberguen variedades diatópicas de menor extensión en su espacio de uso. Tenemos entonces varias dimensiones subordinadas de espacio regional de uso. La concepción de pluricentrismo (Clyne 1991) - que dice que la diferencia de variedades no consiste solamente en el factor espacio, sino que reclama un estatus político (que una variedad diatópica es la variedad estándar de un país) - complica todavía más el concepto de diasistema diatópico. Por ello, el valor político de las diferentes variedades diatópicas no es el mismo. El criterio de la variedad diatópica no es solo espacial, sino que contiene dos criterios. Entra entonces un criterio adicional, el de estatus político.

El concepto de situación comunicativa siempre ha sido pertinente para las otras dimensiones de variación, como definitoriamente de las diafásica y diamésica, pero también de la diastrática. El uso de las variedades del lenguaje popular, ${ }^{9}$ del grupo social de los jóvenes, del caló, del habla de los delincuentes y del lenguaje estándar (este último de uso en el estrato social alto y culto), no solo depende de un factor de pertenencia a un estrato social. Muchos hablantes conocen dos o más variedades diastráticas (por ejemplo, por el ascenso social) y no solo utilizan "la variedad que les corresponde" por ser miembros del estrato o grupo correspondiente, sino que utilizan una variedad considerando la situación social; esto puede servir a una funcionalidad particular; por ejemplo, la gestión de identidad grupal o la expresión de modernidad etc. o para acercarse al interlocutor.

\section{Migración colectiva y situaciones comunicativas bilingüies}

La migración colectiva de un área lingüística hacia otra (no a un área despoblada y sin la exterminación de la población nativa, como pasó en las islas del Caribe, en el sur de Argentina y Chile o en parte de lo que hoy son los EE.UU.) crea situaciones de comunicación entre individuos de las dos lenguas (variedades). Las llamo situaciones de comunicación bilingüe. Las posibilidades de convivencia (o segregación) de los nativos y los migrantes son muy variadas, dependiendo del poder de las poblaciones nativas y migrantes involucradas (invasión militar, imperialismo, colonialismo, migrantes de estatus social bajo o alto, migrantes esclavos, etc.), de ideologías racistas, del motivo de la migración, del establecimiento de instituciones de aprendizaje formal de la otra lengua, de la construcción de identidad, del grado cultural y de la actitud de los nativos, etc. Las consecuencias

\footnotetext{
${ }^{9}$ Empleo este término aquí para simplificar, a pesar de su estatus problemático por la imprecisión del concepto y de connotaciones negativas.
} 
pueden ser la pérdida de una de las dos lenguas. Los migrantes de estatus social bajo pueden dejar de hablar su lengua a lo largo del tiempo tanto como pueden hacerlo los invasores poderosos (como pasó con los invasores germánicos en el imperio romano; los francos, visigodos, lombardos, borgoñeses, suevos, etc. se romanizaron y no impusieron sus lenguas a los pueblos conquistados).

La discusión de lo que pasa en tales casos se focaliza muchas veces sobre la cuestión de si los contactos devienen en la emergencia de una nueva lengua. ${ }^{10}$ Esta perspectiva se debe a una reducción teórica y en algunos casos a una inquietud glotopolítica. Lo que pasa a corto o medio plazo no es la emergencia de una nueva lengua (habrá que definir antes lo que es una "nueva lengua") sino la emergencia de un nuevo diasistema. $\mathrm{Y}$ este incluye varias variedades con rasgos de translingualizaciones (translanguaging). Es esencial considerar que se conforma no solo una sola variedad de contacto, sino varias variedades de las dos lenguas (tanto idiolectales como sociolectales y regiolectales). ${ }^{11}$ Propongo entonces que una meta adicional de los estudios de contacto de lenguas (o de dialectos) debe ser la descripción del diasistema bilingüe de contacto en el espacio de encuentro de hablantes de diferentes lenguas (o dialectos o variedades diatópicas) y su evolución dinámica. Este enfoque abre la visión hacia la diversidad de soluciones individuales y sociales del manejo de lenguas en el contacto y puede detectar con más claridad los procesos de una eventual nivelación y reducción de diversidad en etapas posteriores del contacto hacia una o menos variedades de contacto. Esta meta tiene dos submetas: a) el estudio del manejo cognitivo y variado de las lenguas implicadas a nivel individual y b) la descripción del nuevo diasistema de variedades implicadas que se estabilizan como variedades a nivel grupal.

\section{Heterogeneidad de variedades afrohispanas y variedades en vía de criollización}

Indicios de la existencia de un diasistema de variedades de contacto son los diversos estudios sobre el cómo teorizar la génesis de las lenguas criollas. Se ha enfocado en los años pasados la emergencia de nuevas variedades en los estudios sobre la criollización (o nativización) con un sistema ya estable procedente de variedades pidgin con "sistemas" inestables, o con el language intertwining de

\footnotetext{
${ }^{10}$ Así lo postula Stavans (2003) para el así llamado "spanglish" en los EE.UU, lo que suscitó una larga discusión. Cf. entre otros Otheguy/Stern (2010), López García-Molins (2015). La cuestión abierta es ¿̇cuándo se puede considerar algo como una nueva lengua? Un consenso entre lingüistas es que la novedad de debe manifestar en estructuras gramaticales diferentes. Pero el caso de contactos que devienen en una relexificación alta con el mantenimiento de las estructuras gramaticales no se puede pasar por alto en la discusión.

${ }^{11}$ Zimmermann (1992: 208-210 y 227-230) describe esta heterogeneidad de variedades de contacto en el caso del contacto de las lenguas otomí y español en el Valle del Mezquital de México. Este esquema todavía es simplificador, pero proporciona un perfil individual de la ocurrencia de los rasgos de translingualización de once hablantes.
} 
las lenguas mixtas (cf. Bakker/Mous 1994 entre otros) siempre prevaleciendo el interés por una nueva lengua (considerada como monolítica). Raras veces se ha descrito la gama de variedades de contacto y el diasistema de variedades de contacto emergente. El descubrimiento de variedades que no cumplen con los rasgos básicos para las lenguas criollas (establecidos a base de las lenguas criollas prototípicas) ha llevado a la teoría de un continuo de contacto posterior a la génesis de estos, el de basilectomesolecto-acrolecto (Bickerton 1975). El nivel del mesolecto sería una variedad intermedia que se formaba del contacto del basilecto (la "verdadera lengua criolla", por ejemplo el criollo haitiano) con la adopción de elementos y sobre todo estructuras del acrolecto (el francés), la lengua de la que se tomaba el material léxico en la formación del basilecto, lengua oficial ${ }^{12}$ y estándar de las capas sociales cultas y altas del grupo. ${ }^{13}$ Además, del contacto de lenguas africanas de los esclavos negros con lenguas europeas (en América) surgieron variedades que no cumplen con los criterios estrictos para lo que es una lengua criolla. El análisis de muchos casos de variedades de afrodescendientes que se desarrollaron en condiciones similares a las lenguas criollas en América (papiamentu, palenquero, haitiano, por ejemplo) ha demostrado, sin embargo, muchas formas variadas de como los afrodescendientes manejaron las lenguas africanas y las lenguas española y portuguesa (cf. los estudios acerca de las variedades afrohispanas en Panamá (Lipski 1989) y Bolivia (Lipski 2008), el Valle del Chota (Ecuador) (Sessarego (2013). Para estas se ha propuesto el término de semicriollo, ${ }^{14}$ variedad definida como una entre otras dos con una evolución estancada hacia una lengua criolla. ${ }^{15}$

Disertando sobre el concepto de semicriollo, enfatizado por Holm (2000:10), McWhorter (2014) sugiere que los mesolectos no son el resultado de aproximaciones del basilecto a la lengua estándar (poscriollos) sino un resultado del manejo de lenguas sui generis en el contacto de las lenguas africanas de los esclavos con la lengua del esclavista/colonizador. Podemos considerar que los semicriollos, que ofrecen estructuras bastante diversas entre sí, son testimonio de la pluralidad de variedades resultantes de este tipo de contacto de lenguas. ${ }^{16}$

Para intervenir en los procesos de contacto (favorecer, impedir, frenar, sustituir) se pueden tomar medidas glotopolíticas. La comunidad de hablantes puede menospreciar y estigmatizar las lenguas

\footnotetext{
${ }^{12}$ Fue una imposición política, muchas veces por razones del pasado colonial.

${ }^{13}$ Este tipo de variedades intermedias heterogéneas se ha descrito también en el "contacto" de la lengua estándar y el dialecto regional, por ejemplo en Alemania, denominadas por Auer (2005: 22) variedades diaglósicas conformando cada una un continuo entre las dos lenguas participantes, en vez de variedades discretas en relación diglósica.

${ }^{14}$ Los fenómenos lingüísticos observados que condujeron al término semicriollo son válidos pero el término (que lleva la impronta de la obsesión de encontrar lenguas criollas prototípicas) es desafortunado; sigue-siendo un término controvertido.

${ }^{15}$ Significativa al respecto es también la discusión de si el chamorro (lengua de las Marianas), lengua malayo-polinésica, de la familia austronesia, con un léxico de muy alta cantidad de origen español es una lengua mixta, criolla o semicriolla. Cf. el resumen de las opiniones al respecto en Rodríguez-Ponga (2009: 19-22).

${ }^{16}$ Cabe mencionar (pero no es el tema de este artículo) que no solo elementos de lenguas, estructuras morfológicas y sintácticas, sino lenguas enteras pueden ser afectados, es decir pueden ser sustituidos.
} 
emergidas del contacto, incluso prohibir su uso en ciertos dominios sociales o, al contrario, instituirlas como lenguas nacionales e incluso oficiales. Lo último significa hacerlas objeto de planificación lingüística, como elaboración de léxico, normativización, estandarización, y fomentar e imponer su uso en dominios reservados antes a otra lengua.

En verdad, una variedad se distingue de otra por sus diferencias en los elementos y las estructuras. La correlación de varios de estos rasgos diferentes con factores extralingüísticos (sociales, regionales, estilísticas, identitarias, religiosas etc.) sirve para identificar una variedad tanto por los mismos hablantes como - de manera sistemática y con un trasfondo teórico - por parte de los lingüistas. Por ello, la construcción mental y conceptual de las distintas variedades no es necesariamente la misma. Un ejemplo es el concepto popular y no científico "spanglish". Así, los estudios sobre el contacto a nivel de elementos y estructuras interferidas, transferidas o nuevas hechos por lingüistas son un paso esencial también para el "reconocimiento" de una variedad distinta, pero no es suficiente, sobre todo para los hablantes mismos. Estos construyen su universo de variedades por consideraciones propias en su sistema de cultura lingüística.

\section{Las variedades de los chicanos en EE.UU.}

Un ejemplo de la emergencia de un tal diasistema complejo se ha dado en el caso de la migración de mexicanos en el suroeste de Estados Unidos de América. ${ }^{17}$ Lo hemos esbozado en Zimmermann (2018b) basándonos en una contribución de Ornstein-Galicia (1988).

\subsection{Dos grupos de variedades de contacto en el suroeste de EE.UU.}

En general tenemos que distinguir dos tipos distintos de variedades de contacto teóricamente emergentes por la migración en la diáspora, en cierta forma complementarias:

- $\quad$ Las variedades de contacto (sean idiolectales o sociolectales) basadas en la lengua y variedades del país de origen, en nuestro caso basadas en el español mexicano.

${ }^{17}$ Cf. Sánchez (2010) y Rivera-Mills/Villa (2010) para la situación lingüística del suroeste de EE.UU. 
- $\quad$ Las variedades de contacto (sean idiolectales o sociolectales) basadas en la lengua y variedades del país de llegada, en nuestro caso basadas en el inglés estadounidense del suroeste.

En los EE.UU. se han dado las dos.

Supongo que los dos grupos de variedades han emergido simultáneamente, no el primero antes del segundo, ya que cumplen cada una funciones comunicativas distintas y son estas funciones las que hacen emerger las variedades. El primer grupo de variedades se llama popularmente spanglish; es una variedad utilizada entre los migrantes y los posmigrantes. El término es simplificador ya que no considera la gama de variedades diferentes y pretende ser una sola variedad. Además implica ciertas confusiones ya que este concepto mezcla varios procesos del manejo de dos lenguas que hace falta distinguir. El segundo grupo de variedades es utilizado esencialmente por los migrantes con la población anglohablante. Es una variedad unilateral ya que los hablantes nativos del inglés no la utilizan. Teniendo en cuenta que se trata de un proceso de migración de muchos individuos que arriban en distintos momentos durante un lapso largo de tiempo, no se descarta, sin embargo, la posibilidad de que a nivel individual se adquiera una después de la otra, ya que nuevos migrantes se ven confrontados con las variedades ya existentes entre los migrantes previamente llegados. La primera confrontación con la lengua del país de recepción hace emerger, no obstante, a nivel individual el escenario mencionado.

Las variedades de contacto basadas en la lengua de origen, en nuestro caso el grupo de español chicano, se compone esencialmente del sistema estructural de la lengua materna (el español mexicano) con transferencias $^{18}$ de la lengua del país de llegada (el inglés del suroeste de EE.UU.). Las variedades de contacto basadas en la lengua del país de llegada, las del inglés chicano, constan esencialmente del sistema estructural del inglés con interferencias de la lengua de origen de migración, el español mexicano. En el caso de adquisición urgente y comportamiento lingüístico incontrolado en el momento de la necesidad comunicativa urgente, estos procesos ocurren sin mayores reflexiones normativas de manera inconsciente. Estos hablantes migrantes quieren comunicarse y no se ocupan de lo "correcto" o incorrecto de lo enunciado. ${ }^{19}$

Los estudios de variedades de contacto emergidas por la migración en otros contextos se concentran muchas veces en las variedades basadas en la lengua del país de llegada (por ejemplo migrantes turcos, italianos, españoles y portugueses en Alemania). Esto sería en nuestro caso el inglés

\footnotetext{
${ }^{18}$ Para la definición neurobiológica de los términos transferencia versus interferencia cf. Zimmermann (2019).

${ }^{19}$ Cf. el concepto de translanguaging de Otheguy/ García/ Reid (2015); también los análisis de repertorios lingüísticos en prácticas heterogéneas de lenguas (language repertories in heterogeneous language practices) por Léglise/ Sánchez Moreano (2018).
} 
chicano, pero es de notar que no es así en los estudios de la lingüística de los chicanos; en esta disciplina se privilegian los estudios de las variedades basadas en el español. ¿Se deben estas preferencias de estudio a razones glotopolíticas o simplemente a la identidad científica de los investigadores: germanistas en el caso arriba mencionado o hispanistas en el caso de EE.UU.?

Las variedades de contacto por migración tienen un futuro ambivalente. Algunas se perdieron rápidamente (como el cocoliche de Argentina), otras se mantienen y se convierten en variedades no estándares y otras más en la base de una nueva lengua (el francés emergido de la migración conquistadora de los francos; otro ejemplo: muchas lenguas criollas). El grupo de variedades subsumidos bajo el concepto spanglish se originó desde la primera mitad del siglo XX, sigue hablándose y tiene aparentemente una vitalidad bastante fuerte.

\subsection{El espacio de variedades en la zona y el diasistema del español}

Es importante reconocer que el suroeste de EE.UU. constituye un espacio complejo de lenguas y variedades, resultado de las migraciones sucesivas. Ornstein-Galicia (1988) da un primer paso teórico hacia el reconocimiento de un diasistema y concibe esta situación como un continuo de variedades (ver Figura 1). Lo configura como una cadena de variedades con dos polos opuestos:

Figura 1: Continuo de variedades (según Ornstein-Galicia 1988: 1410)

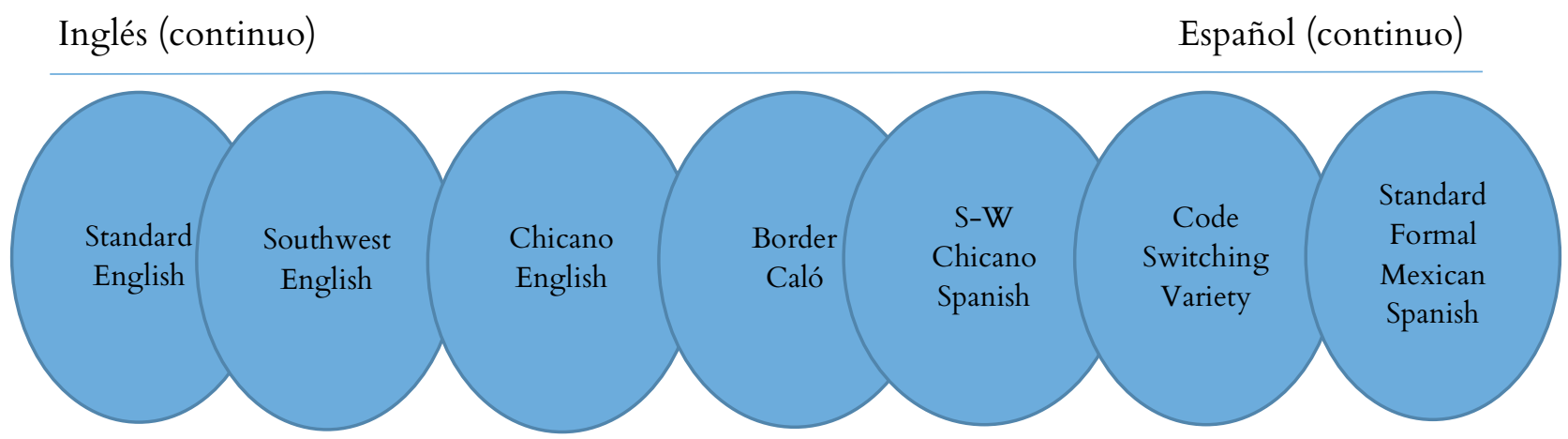

Si se integran otras lenguas y variedades llegamos a una visión más amplia, diferenciada.

El modelo de este espacio, que presento abajo, todavía está, ciertamente, simplificado; habrá que diferenciar aún más si se pretendiera presentar un panorama completo. Del lado de la lengua española 
es importante darse cuenta de que se trata de un espacio diasistémico de variedades donde se cruzan a la vez rasgos subdialectales del español de México ${ }^{20}$ y rasgos de contacto con otra lengua, la inglesa. La multitud de variedades, sin embargo, no debe concebirse como una línea con dos polos sino más bien como un espacio tridimensional. Teniendo en cuenta otros factores llegamos a la siguiente lista en el espacio de variedades en el suroeste de EE.UU. (simplificada).

- Variedades diatópicas, diastráticas y diafásicas del español de México (y variedades centroamericanas) inclusive el español estándar de México.

- $\quad$ Lenguas indígenas mexicanas.

- $\quad$ Variedades del español indígena.

- Variedades diastráticas y diafásicas del inglés de EE.UU. (de los anglohablantes nativos) inclusive el inglés estándar.

- Variedades del español de contacto (producto del translanguaging: "Spanglish"): variedades informales, pachuco (variedades de jóvenes), variedades familiares, ¿variedad formal?

- Las prácticas de la alternancia de lenguas (Code Switching) con varias formas, producto del translanguaging.

- Variedades del inglés de contacto de hablantes de origen mexicano (chicanos) e indígenas mexicanos (variedad étnica o etnolecto del inglés), producto del translanguaging.

- ¿ ¿Una nueva variedad koiné (neutral) del español de EE.UU. (con transferencias del inglés o purificada)?

Cabe añadir que las variedades de contacto mencionadas ya pueden tener un estatus de variedad nativa en las generaciones posmigrantes. Además, no se trata en cada categoría de una variedad, sino de varias expresiones. La presentación en forma de lista renuncia a posicionarlas sobre una línea de contacto con dos polos ya que quiere representar la existencia de variedades en el espacio comunicativo. Hay que tener en cuenta también que este espacio de variedades lleva algunos rasgos de diglosia en el sentido de una valoración social alta y baja según las actitudes de la comunidad

\footnotetext{
${ }^{20}$ Parece que la presencia de otras variedades de otros países hispanohablantes no tienen influencia en las variedades de contacto del español de los chicanos, pero sí pueden ser habladas en ciertas situaciones.
} 
(todavía) mayoritaria, los anglohablantes, con el valor bajo de la dimensión de las variedades con rasgos españoles (Parodi 2011). Se puede observar que parte de la población chicana, la que quiere asimilarse culturalmente, comparte esta valoración.

Como-puede reconocerse hay que distinguir aquí una perspectiva social y otra individual. Desde la primera perspectiva podemos decir que las variedades están presentes en el área. El conocimiento y el uso de las variedades a nivel individual es distinto; no todos los individuos dominan y utilizan de igual manera estas variedades. Algunos son monolingües, otros bilingües, los migrantes indígenas son trilingües y su repertorio variacional tiene esta dimensión adicional; otros pueden ser además hablantes multidialectales. Los bilingües chicanos dominan (en grados de perfección diferente) el Western English, en parte el Southern English, el inglés chicano que se caracteriza en gran parte de interferencias del español ${ }^{21}$, el español mexicano o uno de sus variedades regionales, una variedad del español chicano o spanglish chicano con transferencias del inglés ${ }^{22}$, el caló fronterizo y, como práctica vastamente aceptada, la alternancia de código. Parte de los jóvenes chicanos desarrollaron un lenguaje juvenil (el pachuco) o varios otros. A nivel de la recepción, los hispanos del suroeste están también expuestos al español mexicano estándar en los medios de comunicación; se puede suponer que muchos entienden esta variedad. Los egresados de formación universitaria como maestros, los empleados de los medios masivos y los profesionales sin duda dominan las variedades estándar del inglés activamente, algunos posiblemente con acento español. Durante los contactos intensos y frecuentes entre los grupos étnicos se producen influencias recíprocas de las diferentes variedades del español a nivel individual.

\section{Repensar el marco teórico del diasistema}

Las formulaciones del diasistema mencionadas arriba tienen la pretensión de validez universal. El número de variedades que estaba desarrollando la comunidad chicana (si se incluyen los indígenas mexicanos bilingües a esta comunidad) ${ }^{23}$ es mayor que el de una comunidad monolingüe y con ello más complejo. Sin embargo, parece que el uso de las variedades encontradas en la comunidad chicana

\footnotetext{
${ }^{21}$ Por razones de espacio no puedo presentar las características del inglés chicano en el marco de este artículo. Algunas se presentan en Ornstein-Galicia (1988: 1410); otras en Fought (2003). En la página web de la University of Portland se encuentra una lista bien explicada para un público no especialista, (http://www.pdx.edu/multicultural-topicscommunication-sciences-disorders/chicano-english).

${ }^{22}$ Por razones de espacio no puedo presentar las características del español chicano en el marco de este artículo, cf. Parodi (2011, 2014) y Zimmermann (2018a).

${ }^{23}$ Queda por investigar por la sociología si se puede de veras hablar de una comunidad chicana, si por ejemplo los indígenas mexicanos bilingües forman parte de la comunidad chicana, es decir si hay comunicación entre ellos.
} 
se puede atribuir a los criterios establecidos, espacio, pertenencia social (si se incluye el factor étnico), situación y medios de comunicación.

La idea del diasistema fue elaborada como arquitectura de variedades de una lengua (histórica) con sus dialectos primarios tradicionales y otras variedades regionales o locales al nivel diatópico. La cuestión de si un dialecto forma parte de la lengua histórica u otra lengua con una propia diferenciación interior es asunto de evaluación de lingüistas y también de declaraciones políticas (como es bien conocido del caso del catalán y valenciano o del bajo alemán con referencia al alto alemán). Como se sabe, pero se olvida a veces, los dialectos tampoco son homogéneos y tienen diferenciación diastrática, diafásica y a veces diamésica interna; por ejemplo en la emergencia de variedades mixtas del dialecto y la lengua estándar. ${ }^{24}$

El concepto de diasistema se basa en la lengua. Esta visión hace suponer que en el caso de comunidades de migración y de emergencia de variedades étnicas ${ }^{25}$ (con translingualizaciones) los migrantes afectados viven en dos diasistemas, en el caso de los chicanos el del español mexicano y el del inglés estadounidense, perteneciendo cada variedad con sus rasgos de translingualización a uno de los dos diasistemas. Como hemos visto, esto no es así. En vez de un paralelismo de dos diasistemas se ha desarrollado un diasistema híbrido con nuevas variedades cuya atribución a una lengua no es clara; depende de criterios más o menos consensualmente establecidos por la lingüística: gramaticales versus lexicales. La validez del criterio aplicado se debe a una preferencia teórica: Si la gramática de variedades híbridas obedece fundamentalmente a una lengua, entonces cuenta como una variedad de esta, no importa el aumento de elementos lexicales de otra lengua. Sea cual sea el criterio justo o adecuado (tal vez no hay una decisión final objetiva), se trata de una o más variedades nuevas que tienen un estatus comunicativo, de prestigio e identidario propio que vale para toda la comunidad de comunicación. Y esta incluye a los hablantes monolingües del inglés.

Podemos preguntarnos, si los grupos o comunidades de migración viven en dos diasistemas lingüísticos que se traslapan en algunas esferas o hay que suponer un nuevo diasistema reorganizado (construido por dos diasistemas antes separados) que constituye un repertorio de variedades a disposición de la comunidad. Para entender adecuadamente lo que se ha desarrollado en el contacto no sería entonces lo esencial la atribución de pertenencia de una variedad a un diasistema de una lengua o de otra, de manera disyuntiva ${ }^{26}$, sino la práctica de hibridación lingüística de la comunidad de hablantes. El concepto adecuado para dar cabida a esta situación es el de espacio sociocomunicativo. Y

\footnotetext{
${ }^{24}$ En Alemania hay un uso diferenciado del dialecto en una situación y la variedad mixta en otra más formal.

${ }^{25}$ Las variedades étnicas, por ejemplo el inglés chicano, las variedades del español indígena en Hispanoamérica, tampoco caben en la concepción tradicional del diasistema. No deben equipararse con el factor social ni situacional, sino que llevan rasgos de ambos y por ello constituyen una dimensión aparte.

${ }^{26}$ Lo que no hace superfluo la identificación de los microprocesos en términos de la lingüística, cf. Zimmermann 2019).
} 
este espacio consiste de varios subespacios sociocomunicativos propios. Se ha desarrollado así un diasistema de varios subespacios sociocomunicativos: entre los chicanos por un lado, de los chicanos con la comunidad de anglófonos no chicanos, por el otro. Y estos dos consisten en subespacios todavía más particulares, dependiendo de situaciones sociales e institucionales más concretas (escuela, universidad, religión, lugar de trabajo, comercio, medios masivos, familia etc.).

\section{Conclusión y tareas futuras}

La discusión de si una variedad de contacto (en nuestro ejemplo el "spanglish") es una nueva lengua se basa en una teoría inadecuada de lengua y dirige el foco de análisis diacrónico y el pronóstico del desarrollo futuro hacia un constructo anticuado de lengua monolítica. Y con ello conduce a una discusión innecesariamente polémica. Al contrario, debemos considerar el desarrollo como una amplificación y con ello una reestructuración del diasistema de una parte de la población. Este diasistema es nuevo, no una lengua nueva monolítica.

A nivel teórico de lengua como diasistema hemos detectado un problema general: La concepción del diasistema como arquitectura de una lengua no es válida para comunidades bilingües. La comunidad de comunicación no se ha percibido como factor de análisis en el caso de comunidades monolingües por su presencia normal, inadvertida porque era implícita en el concepto de lengua, cuasi "naturalmente" dada. Este factor, sin embargo, debe considerarse explícitamente como el factor supremo que define el marco de uso de las variedades lingüísticas. Las comunidades monolingües serían entonces un caso (particular) de comunidades de comunicación entre otros.

Así las cosas, surgen algunas tareas para concretizar la verdadera arquitectura de situaciones de este nuevo espacio sociocomunicativo. Hay que definir las situaciones sociocomunicativas vigentes para poder encontrar el correlato social y situacional en el uso de una u otra variedad. Algunas ya están descritas, como por ejemplo la familia ${ }^{27}$ y el uso de la o las variedades en este subespacio.

Otro aspecto abierto es la cuestión de si se puede incluir en el espacio comunicativo de los chicanos a los indígenas mexicanos bilingües, muchas veces de migración temporal, o si estos constituyen espacios sociocomunicativos propios.

Además, en este marco teórico deben diferenciarse la competencia de las variedades a nivel productivo (activo) y a nivel de comprensión receptiva.

\footnotetext{
${ }^{27}$ Pero debemos prever que tampoco la comunicación familiar se maneja igual en todas las familias, ya que hay familias étnicamente homogéneas y otras que pueden ser constituidas por matrimonios mixtos; además puede tener un impacto la presencia de abuelos y, evidentemente, el grado de educación y la construcción de identidad.
} 


\section{Referencias bibliográficas}

ARGENTE, Joan A. Contactos entre lenguas y sus consecuencias. In: HOLTUS, Günter; METZELTIN, Michael; SCHMITT, Christian (eds.). Lexikon der romanistischen Linguistik. vol. VII. Tübingen: Niemeyer, 1998. p. 1-14.

AUER, Peter. Europe's sociolinguistic unity, or: A typology of European dialect/standard constellations. In: DELBECQUE, Nicole; VAN DER AUWERA, Johan; GEERAERTS, Dirk (eds.). Perspectives on Variation: Sociolinguistic, Historical, Comparative. Berlin: De Gruyter, 2005. p. 7-42.

BAKKER, Peter; MOUS, Maarten (eds.). Mixed Languages: 15 Case Studies in Language Intertwining. Amsterdam: IFOTT.

BERNAL-ENRÍQUEZ, Ysaura. La sociohistoria de la pérdida del español en la Nueva México. In: ORTIZ LÓPEZ, Luis A.; LACORTE, Manel (eds.). Contactos y contextos lingüísticos: el español en los Estados Unidos y en contacto con otras lenguas. Madrid/Frankfurt, Iberoamericana/Vervuert, 2005. p. 271-285.

BICKERTON, Derek. Dynamics of a Creole System. Cambridge: Cambridge University Press, 1975.

BILLS, Garland D. Las comunidades lingüísticas y el mantenimiento del español en Estados Unidos. In: ORTIZ LÓPEZ, Luis A.; LACORTE, Manel (eds.). Contacto y contextos lingüísticos: el español en los Estados Unidos y en contacto con otras lenguas. Madrid/ Frankfurt am Main: Iberoamericana/Vervuert, 2005. p. 55-83.

CLYNE; Michael (ed.). Pluricentric Languages: Differing Norms in Different Nations. Berlin: de Gruyter Mouton, 1991.

COSERIU, Eugenio. Lecciones de lingüística general. Madrid: Gredos, 1981.

COSERIU, Eugenio. Historische Sprache und Dialekt. In: ALBRECHT, Jörn; LÜDTKE, Jens; THUN, Harald (eds.). Energeia und Ergon. Sprachliche Variation, Sprachgeschichte, Sprachtypologie. Studia in honorem E. Coseriu. Vol. 1. Tübingen: Narr, 1988. p. 54-61.

FOUGHT, Carmen. Chicano English in Context. New York: Palgrave Macmillan, 2003.

HALLIDAY, Michael A.K. Language as Social Semiotic: The Social Interpretation of Language and Meaning. London: Arnold, 1978.

HOLM, John. An Introduction to Pidgins and Creoles. Cambridge: Cambridge University Press, 2000.

KELLER, Rudi. Sprachwandel. Von der unsichtbaren Hand in der Sprache. 3a ed. corr. Tübingen/Basel: Francke, 2003. 
KOCH, Peter; OESTERREICHER, Wulf. Sprache der Nähe - Sprache der Distanz. Mündlichkeit und Schriftlichkeit im Spannungsfeld von Sprachtheorie und Sprachgeschichte, Romanistisches Jahrbuch 36, p. 15-43, 1985.

LÉGLISE, Isabelle; SÁNCHEZ MOREANO, Santiago. From varieties in contact to the selection of linguistic resources in multilingual settings. In: BASSIOUNEY, Reem. (ed.). Identity and Dialect Performance: A Study of Communities and Dialects. London/ New York: Routledge, 2018.p. 143-159.

LIPSKI, John. The Speech of the Negros Congos of Panama: a Vestigial Afro-Hispanic Creole. Amsterdam: Benjamins, 1989.

LIPSKI, John. Afro-Bolivian Spanish. Frankfurt and Madrid: Vervuert/Iberoamericana, 2008.

LOPE BLANCH, Juan M. (dir.). Atlas lingüístico de México. 3 vols. México: El Colegio de México, 1990-1992.

LÓPEZ GARCÍA-MOLINS, Ángel. Teoría del Spanglish. Valencia: Tirant Humanidades, 2015.

MCGREGOR-MENDOZA, Patricia. El desplazamiento intergeneracional del español en los Estados Unidos: una aproximación. In: ORTIZ LÓPEZ, Luis A.; LACORTE, Manel (eds.). Contactos y contextos lingüísticos: el español en los Estados Unidos y en contacto con otras lenguas. Madrid/Frankfurt, Iberoamericana/Vervuert, 2005. p. 287-300.

MCWHORTER, John. Mesolect as the norm: semi-creoles revisited, Papia, 24 (1), p. 7-25, 2014.

MEJÍAS, Hugo A.; ANDERSON-MEJÍAS, Pamela L.; CARLSON, Ralph. Attitude Update: Spanish on the South Texas Border, Hispania, 86.1, p. 138-150, 2003.

OTHEGUY, Ricardo; STERN, Nancy. On so-called Spanglish, International Journal of Bilingualism, 15: 1, p. 85-100, 2010.

OTHEGUY, Ricardo; GARCÍA, Ofelia; REID, Wallis. Clarifying translanguaging and deconstructing named languages: A perspective from linguistics, Applied Linguistics Review 6: 3, p. 281-307, 2015.

ORNSTEIN-GALICIA, Jacob. Form and Function in Chicano English. Rowley, Mass.: Newbury House, 1985.

ORNSTEIN-GALICIA, Jacob. The U.S. Southwest as a sociolinguistic problem area. In: AMMON, Ulrich; DITTMAR, Norbert; MATTHEIER, Klaus (eds.). Sociolinguistics. An International Handbook of Language and Society. Berlin, de Gruyter, 1988. p. 1405-1414. PARODI, Claudia. El otro México: español chicano, koineización y diglosia en Los Ángeles, California. In: MARTÍN BUTRAGUEÑO, Pedro (ed.). Realismo en el análisis de corpus orales (primer coloquio de cambio y variación lingüística. México: El Colegio de México, 2011. p. 217-243. 
PARODI, Claudia. El español y las lenguas indígenas de los mexicanos en los Estados Unidos. In: BARRIGA VILLANUEVA, Rebeca; MARTÍN BUTRAGUEÑO, Pedro (eds.). Historia sociolingüística de México, vol. 3, Espacio, contacto y discurso político. México: El Colegio de México, 2014. p. 1525-1567.

RIVERA-MILLS, Susana V.; VILLA, Daniel J. (eds.). Spanish in the U.S. Southwest: A Language in Transition. Madrid/Frankfurt: Iberoamericana/Vervuert, 2010.

RODRÍGUEZ-PONGA, Rafael. Del español al chamorro. Lenguas en contacto en el Pacífico. Madrid: Ediciones Gondo, 2009.

SÁNCHEZ, Rosaura. Mapping the Spanish language along the multiethnic and multilingual border. In: NORIEGA, Chon A.; ÁVILA, Eric; DÁVALOS, Karen Mary (eds.). The Chicano Studies Reader. Los Angeles, Ca., University of California, 2010. p. 511-566.

SESSAREGO, Sandro. Chota Valley Spanish. Madrid/Frankfurt: Iberoamericana/ Vervuert, 2013.

STAVANS, Ilan. Spanglish: The Making of a New American Language. New York, HarperCollins, 2003.

US Census Bureau 2010, http://www.census.gov/2010census/.

WEINREICH, Uriel. Languages in Contact. Findings and Problems. New York: Publications of the Linguistic Circle of New York, 1953.

ZIMMERMANN, Klaus. Sprachkontakt, ethnische Identität und Identitätsbeschädigung. Aspekte der Assimilation der Otomí-Indianer an die hispanophone mexikanische Kultur. Frankfurt am Main: Vervuert, 1992.

ZIMMERMANN, Klaus. Migración, contactos y nuevas variedades lingüísticas: Reflexiones teóricas y ejemplos de casos de América Latina. In: ESCOBAR, Anna María; WÖLCK, Wolfgang (eds.). Contacto lingüístico y la emergencia de variantes y variedades lingüísticas. Frankfurt am Main: Vervuert/ Madrid: Iberoamericana, 2009. p. 129-160.

ZIMMERMANN, Klaus. La frontière linguistique hispano-anglaise entre le Mexique et les ÉtatsUnis d'Amérique. In: OSSENKOP, Christina; WINKELMANN, Otto (eds.). Manuel des frontières linguistiques dans la Romania. Berlin/Boston: de Gruyter, 2018a. p. 476-501.

ZIMMERMANN, Klaus. Mantenimiento y pérdida de variedades de contacto emergidas de la migración: La dinámica de los diasistemas de los chicanos. In: PATZELT, Carolin; SPIEGEL, Carolina; MUTZ, Katrin (eds.). Migración y contacto de lenguas en el siglo XXI. Berlin et al.: Peter Lang, 2018b. p. 21-40.

ZIMMERMANN. Klaus. La translingualización como resultado del manejo de las lenguas en situaciones de contacto: la perspectiva del constructivismo neurobiológico. In: HABOUD, Marleen (ed.). Lenguas en contacto: desafíos en la diversidad. Quito: PUCE, 2019. p. 4779. 